\title{
SUR QUELQUES MASTIGAMIBES DES ENVIRONS DE GENĖVE
}

$\mathrm{P} \wedge \mathrm{R}$

\section{E. PENARD}

Avec les planches 10 et 11 .

Les Protozoaires qui font l'objet du mémoire actuel sont des Amibes, qui se comportent absolument comme les autres représentants de ce groupe, et pourtant aussi des Flagellates, car ils possèdent un fouet, mince, délicat, parfois rudimentaire et souvent inutile, mais un fouet véritable, un flagelle, muni de son grain basal caractéristique.

Ces organismes, que l'on réunit aujourd'hui en un ordre spécial, celui des Mastigamibes (GoLdschmidt $1907^{1}$ ), forment un petit groupe dans lequel GoLDSCHMIDT distingue 17 espèces, parmi lesquelles, il est vrai, on en pourrait citer plusieurs qui sont bien peu connues encore.

Dans le cours de mes études générales sur les Rhizopodes, et dès l'année 1902, j'ai eu de temps à autre l'occasion d'examiner quelques-uns de ces organismes; mais ce n'est que cette année méme, en 1909, qu'après avoir pu suivre tout au long trois au moins des représentants de ce groupe, je me suis cru autorisé à

${ }^{1}$ Ce sont là, pro parte, les Rhizoflagellates de S. Kent (188), Manual of the Infusoria), Rhizomastigina de BüTschli (1883, Bronn's Tierreich). On trouvera l'historique de la question, comme aussi une liste bibliographique, dans l'excellent travail de GoLdschmidt (5). 
reprendre mes notes et mes anciens croquis, pour réunir en un tout homogène les observations que j'ai pu faire à ce sujet.

Mastigameba aspera F.-E. Schulze.

Syn. Dinamoba mirabilis Leidy.

(Pl. 10, Fig. 1 et 2.)

En $1875^{1}$, F.-E. Schulze décrivait sa Mastigamoba aspera; mais un peu avant cette époque déjà, en $1874^{2}$, LEIDY s'était occupé d'une "Amibe», Deinamceba mirabilis, que plus tard, en 1879 (7), il décrit plus au long, cette fois sous le nom quelque peu modifié de Dinamoeba mirabilis, et qu'il donne comme ressemblant fort à la Mastigamoba de ScHulzE.

Depuis lors, de différents côtés sont venus des renseignements sur ces deux organismes; Archer (1), Frenzel (4), Schneider (11), Goldschmidt (5), Cash (3), etc., ont rencontré la Mastigamoba, Leidy (7), Greeff (6), Blochmann (2), Penard (8), et quelques autres encore, ont étudié la Dinamœba; mais aucun de ces observateurs ne semble avoir eu l'occasion d'examiner l'une et l'autre de ces deux formes.

Tous, par contre, après des études poussées souvent très à fond, en arrivent à la même conclusion : ces deux organismes présentent entre eux une analogie remarquable, si remarquable même, qu'au premier abord on est tenté de les assimiler l'un à l'autre; taille, forme, apparence, structure, pseudopodes avec leurs caractères si particuliers, noyaux, vésicules contractiles, plasma, aspérités caractéristiques qui ne se retrouvent nulle part ailleurs, tout y est, et y est identique; et pourtant l'un possède un flagelle et l'autre ne l'a pas! la Mastigamoba est un Flagellate, la Dinamerba une Amibe.

${ }^{1}$ Archiv f. mikrosk. Anatomie. V. 11, 1875, p. 583.

${ }^{2}$ Proceed. Acad. Nat. Sc. Philad. 1874, pp. 142, 155. 
Dans la Dinamoba, il est vrai, on pouvait supposer l'existence d'un flagelle, qui aurait échappé à l'attention des naturalistes, mais après les recherches très minutieuses de certains de ces observateurs, la chose restait à peine croyable. Voici par exemple ce que Frenzel (4) dit à ce sujet : «..... Andererseits hatte LEIDY von New-Jersey her einen Organismus als Dinamoba mirabilis beschrieben, welcher so wohl mit dem oben erwähnten (Mastigamoba aspera), als auch mit dem unteren (la Mastigamaba Schulzei de FrenzeL, très voisine de la précédente) in der Konfiguration eine auffallende Aehnlichkeit hat. Nur trägt er keine Geissel, und zwar gibt dies LEIDY, der ein so trefflicher Beobachter war, mit grösster Bestimmtheit an, indem er hervorhebt, wie er, selbst stutzig gemacht, bei einer erneuerten Untersuchung ganz besonders auf diesen Umstand geachtet habe. Wenn bei diesen Rhizopoden eine Geissel vorhanden, so fällt sie meist sofort in die Augen und kann nicht gut übersehen werden, wesshalb mir ein Zweifel an den Angaben LEIDY's nicht angebracht erscheint. »

GReEFF (1888) s'exprime dans des termes presque identiques, et GoLDSCHMidT est tout aussi affirmatif : "Was endlich die Homologisierung mit LEIDY's Dinamaba mirabilis betrifft, die SCHNEIDER ebenfalls durchführen möchte, so ist sie auch ausgeschlossen, da Blochmann (1894), wie Penard (1902), die Form wieder gesehen haben und übereinstimmend mit LEIDY (1879) schildern. (Blochmann fand, dass zwei Kerne vorhanden waren ${ }^{1}$.) Mastigamöbencharakter wäre diesen beiden Forschern sicher nicht entgangen. »

Et pourtant, cette identité existe! la Dinamoba mirabilis de LEIDY n'est pas autre chose que la Mastigamoba aspera de SchulzE! Je crois pouvoir en donner la preuve aujourd'hui.

Il y a longtemps, du reste, que pour mon compte, je considérais

1 J'avais, moi aussi, parlé de deux noyaux; le fait a échappé à GoLdschmidt. 
cette identité comme à peu près certaine; il me paraissait presque impossible que deux êtres semblables (à part, naturellement, la question du flagelle) jusque dans leurs moindres détails — détails ici tout particulièrement nombreux et caractéristiques - pussent appartenir à deux classes toutes différentes; mais, n'ayant jamais eu l'occasion d'observer autre chose que la forme Dinamoba, je ne pouvais m'en tenir qu'aux suppositions.

L'année dernière, cependant, en 1908, retrouvant la Dinamoba mirabilis au marais de Feuillasse, mon attention fut dès le premier jour attirée par des mouvements très légers et étranges qui se produisaient sur certains individus; l'animal semblait, sans raison, comme secoué, pivotant quelque peu sur lui-même. Ces mouvements, du reste, je me rappelais les avoir observés déjà, les années précédentes, mais en les attribuant à des Bacilles, ou Flagellates extraordinairement petits qui se seraient lancés sur l'Amibe; mais cette fois je les étudiai plus attentivement il n'y avait pas de trace de.Microbes.

Plus tard, une Amibe se montra, puis une autre, une troisième et d'autres encore, qui toutes revêtaient les caractères de la Dinamoba mirabilis, mais telle qu'on aurait pu la supposer * jeune», plus petite, plus claire, plus remuante, et ces petits individus nageaient alors, avec une certaine rapidité même, au moyen d'un flagelle antérieur que je finis par découvrir dans chacun des exemplaires examinés.

Je me rappelai alors que la Dinamœba m'avait quelquefois paru «nager»; une de mes notes, de 1901, portait ces mots : «Peut aller assez vite, bien que les pseudopodes n'y soient pour rien »; et dans une autre, on lisait: "Cet organisme, en général très paresseux, peut, lorsqu'il le veut, marcher très vite; il est posé sur ses pseudopodes, et marche, ou nage presque, en tournoyant un peu; mais quand on examine les pointes des pseudopodes, on les trouve absolument immobiles. »

Continuant alors mes investigations, concentrant toute mon 
attention sur la périphérie de chaque individu, petit ou gros, que je rencontrais, ou bien isolant ces individus dans une goutte d'eau claire et les laissant se comprimer peu à peu sous la pression du couvre-objet, je finis par trouver le flagelle, l'étudier, le voir battre, le suivre dans les différentes évolutions, sur les trois quarts des individus, même souvent sur les plus gros (qui néanmoins en sont quelquefois certainement dépourvus).

Tout cela, c'était bien la Mastigamoba aspera de Schulze, on n'en pouvait douter; mais c'était en même temps, et d'une manière tout aussi évidente, la Dinamœba mirabilis de LEIDY, cette fois pourvue d'un flagelle.

Bref, après une étude minutieuse, reprise et contrólée au printemps, puis à l'été de 1909, je suis arrivé à la conclusion, qui pour moi est formelle, que Mastigamoba et Dinamoba ne font qu'un.

Mais alors, comment s'expliquer le fait, extraordinaire semble-t-il, que le flagelle ait échappé à des observateurs comme Leidy, Greeff, Blochmann, qui concentraient leur attention sur ce point même?

A mon avis, on peut faire à ce sujet les deux suppositions suivantes :

$1^{\circ}$ La Dinamoba n'est autre qu'une Mastigamoba-dont le fouet se serait perdu, perdu même peut-être sur tous les individus pris dans telle ou telle station, à l'exclusion d'une autre où on le trouverait encore.

$2^{\circ}$ Ce pourrait être une Mastigamoba dans laquelle le flagellum serait d'une délicatesse et d'une transparence telles, qu'il aurait passé jusqu'ici inaperçu.

La première de ces suppositions semble tout d'abord être la plus naturelle, et par elle-même, elle n'est pas sans exercer un certain attrait. Il n'est pas douteux que, dans certaines stations tout au moins, on trouve des individus absolument dépourvus de flagelle, parmi les autres qui en ont; soit que ces individus n'en 
aient jamais possédé, soit plutôt, si l'on a égard au fait que cette absence de fouet est bien plus fréquente chez les adultes que chez les jeunes, qu'ils l'aient perdu au cours de leur existence. Le flagelle, en effet, si j'ai bien observé, est surtout un organe de jeunesse; suffisamment vigoureux, sur les individus de taille très faible, pour jouer un rôle locomoteur évident, il devient à peu près inutile aux adultes; il reste tel qu'il a été, ne s'allonge ni ne grossit, se montre très paresseux, et semble, comme FrEnzed l'a déjà remarqué, "servir plutôt comme organe tactile que comme appareil locomoteur ». Il est devenu, en somme, inutile, et de là à supposer qu'il disparaisse avec l'âge, il n'y a qu'un pas ${ }^{1}$.

De plus, si nous prenons en considération le fait, très vraisemblable comme on le verra bientôt, que dans certaines stations le flagelle est beaucoup plus délicat, moins visible que dans d'autres, rien n'empêche de supposer qu'il y a là un organe rudimentaire, destiné à disparaître, et qui, dans telle ou telle localité, aurait disparu déjà.

Mais, tout en reconnaissant la valeur possible de cette explication, je pense que la seconde hypothèse est plus fondée, et que, dans la plupart-des cas au moins, le flagelle, bien qu'existant, n’a simplement pas été aperçu.

On·a vvu plus haut que, en 1900 déjà, en étudiant la Dinamœba, j'avais constaté parfois de légers tremblotements du corps tout entier, ou bien un mouvement de progression qui rappelait plutôt la nage, sans que les pseudopodes y fussent pour rien; or, c'étaient là des Mastigamœba, puisque nous savons aujourd'hui que les deux noms ne font qu'un; ces mouvements étranges étaient alors bien sûrement causés par un flagelle, qui pourtant n'a pas été vu. Un autre fait également rentre dans le même

\footnotetext{
${ }^{1} \mathrm{~J}$ 'ai fait, à cet égard, certaines expériences relatives à la disparition brusque du flagelle, après compression ou lésion artificielle de l'animal, et les résultats en ont été identiques à ceux que m'a fournis la Mastigella nitens, sur laquelle ces expériences seront relatées en leur temps.
} 
ordre d'idées: dans la Mastigamoba, le flagelle est fixé sur la membrane nucléaire, et cette membrane se voit souvent déformée, étirée, apparence toute spéciale, due à la traction même du flagelle; or, cette apparence, je l'avais remarquée, lors de mes études sur la Dinamœba mirabilis, mais sans pouvoir la comprendre; aujourd'hui, tout s'explique: un flagelle invisible tirait sur l'enveloppe nucléaire très souple.

Il est possible, alors, que d'autres aussi s'y soient laissé tromper, que ni LeIdy, ni Blochmann, ni GreefF n'aient distingué l'organe locomoteur; et pourquoi? C'est ici que nous arrivons à la seconde hypothèse: le flagelle est plus ou moins délicat, plus ou moins visible, suivant la localité d'où proviennent les individus; dans les cas extrêmes, on ne l'aperçoit qu'avec la plus grande difficulté, ou seulement si l'on est prévenu.

A mon avis, telle est là l'explication la plus plausible, mais pour l'étayer, ce n'est, ici encore, qu'à moi-même que je puis m'en référer.

En premier lieu, il est à remarquer qu'aucun des observateurs qui se sont occupés de la Mastigamoba n'indique le flagellum comme très particulièrement difficile à distinguer; ils le donnent comme fin, très clair, souvent peu net à la vue, mais enfin définissable sans trop de peine; FrEnzEL s'exprime même en ces termes: "Wenn bei diesen Rhizopoden eine Geissel vorhanden, so « fällt sie meist sofort in die Augen und kann nicht gut übersehen «werden ». Dans la localité où j’ai étudié cette Mastigamibe, je puis assurer que le flagelle s'est toujours montré d'une finesse extraordinaire; ou plutôt, faudrait-il dire, ce flagelle possédait un indice de réfraction tellement rapproché de celui du liquide ambiant, qu'il passait inaperçu; pour l'étudier, il fallait, une fois l'animal trouvé, chercher le flagelle avec un grossissement relativement faible (150 diamètres) et en diaphragmant fort - une sorte d'ultra-microscopie presque indispensable alors - puis, une fois trouvée la région où s'insérait le flagelle, mettre au 
point sur cette région même, avec le grossissement plus fort; l'œil fixé sur ce point unique trouvait alors le flagellum, avec son épaisseur véritable, son grain basal, etc. Mais combien de fois, même sur des individus isolés, comprimés, et qui se présentaient dans d'excellentes conditions, l'ai-je encore perdu de vue, pour le rechercher avec le faible objectif et le trouver de nouveau!

Et pourtant, c'était avec ce même microscope que dans les années précédentes j'avais étudié les aspérités ou granulations caractéristiques qui recouvrent les pseudopodes, que je les avais vues se dédoubler, et qu'il m'avait été possible de prouver leur nature en tant que Bacilles véritables, une question qui n'avait pas été résolue jusque là; et ces grains caractéristiques, ils étaient aussi petits, plus petits même, que ne l'avaient indiqué les auteurs !

Tous les observateurs, en définitive, semblent avoir considéré l'étude des grains caractéristiques comme plus difficile que celle des flagelles; et dans mon cas particulier, c'était le contraire qui était vrai.

Quoi qu'il en soit, Dinamibe et Mastigamibe ne font qu'un; et si j'ai cru devoir consacrer un temps un peu long à démontrer cette identité, c'est qu'il y a là une question d'une réelle importance. Nous nous trouvons, en effet, en présence d'un organisme qui, par toute sa physiologie comme par sa structure, se rattache nettement aux Rhizopodes; bien que Flagellate en fait, il n'a plus de cette classe, à l'âge adulte au moins, qu'un flagelle à peu près inutile, un organe pour ainsi dire rudimentaire; c'est un Flagellé en train de devenir Rhizopode, ou un Rhizopode qui conserve des souvenirs d'un ancien état flagellé.

Avant de passer à l'étude des quelques autres organismes du même groupe qu'il nous reste à considérer, je voudrais, sans m'occuper autrement de la Mastigamoba aspera dans ses traits généraux, consacrer encore quelques lignes à certains points spéciaux ayant rapport à cet organisme : 
Dans le genre Mastigamœba, le flagelle s'insère sur le noyau, auquel il est solidement attaché par un renflement spécial, ce « grain basal » caractéristique de tous les Flagellates en général, et sur la nature duquel on n'est pas encore au clair. Bien souvent, si l'on examine avec attention l'animal en marche, on voit le noyau, à sa partie antérieure, revêtir une forme quelque peu extraordinaire, plus ou moins étirée, et quelquefois nettement conique, comme l'indiquent à la $\mathrm{Pl}$. 10, les figures 1 et 2 , ou cet étirement caractéristique est tout particulièrement prononcé ${ }^{1}$.

Cet étirement ne peut sans aucun doute être dû qu'à l'action du flagelle même, et nous ne savons alors ce qui doit nous frapper le plus, de l'intensité de l'effet produit par un organe que nous avons reconnu pour être si peu efficace en tant qu'appareil locomoteur, ou de la plasticité remarquable de la membrane nucléaire. Il faut noter aussi que de son côté le nucléole, qui, la plupart du temps, garde sa forme sphérique (fig. 2), peut d'autres fois se déformer et s'étirer lui aussi (fig. 1). On pourrait croire que le nucléole lui-même est en rapport avec le flagelle, au moyen d'un filament extraordinairement fin qui traverserait le suc nucléaire (ce serait alors le Rhizoplaste qu'on a décrit dans certains Flagellates supérieurs?) et positivement, dans un des exemplaires examinés, j'ai cru voir une fine traînée de plasma (représentée en pointillé dans la figure 1). Mais je n'oserais insister sur cette observation; c'est à la limite de la visibilité microscopique que les erreurs subjectives sont le plus faciles, et peut-être, dans le cas actuel, n'y avait-il rien, là où j'ai cru voir quelque chose.

Ajoutons encore que, sauf dans les cas assez rares d'une marche active, pendant laquelle la partie antérieure de l'animal

${ }^{1}$ Dans la fig. 2, l'étirement est même si prononcé, que la membrane nucléaire fait, ou semble faire, saillie dans le milieu ambiant. 
s'allonge et s'étire, l'élément nucléaire ne se voit presque jamais, caché qu'il est sous les particules de différente nature, grains d'excrétion, etc., qui remplissent le corps; aussi la plupart des auteurs ne parlent-ils guère du noyau, et LEIDY, par exemple, ne l'a pas vu, "sauf peut-être », dit-il, "sur des individus jeunes. »

Cependant, si l'on isole l'individu et qu'on le laisse s'étaler peu à peu sous la pression du couvre-objet et par évaporation lente de l'eau, on met facilement cet appareil nucléaire en évidence; mais alors, ce n'est pas un noyau qu'on a sous les yeux, mais bien deux, ni plus ni moins; la Mastigamcba aspera est un organisme binucléé.

Il est étrange que, à l'exception de Blochmann, qui, en 1894, a vu normalement deux noyaux, et de moi-même qui, en 1902, arrivais aux mêmes conclusions, on n'ait jamais parlé que d'un seul de ces organes. Frenzel (à propos de sa Mastigamoba Schulzei, presque identique à la $M$. aspera), n'a trouvé, dit-il, qu'un noyau, sauf dans deux cas exceptionnels où il y en avait deux. En réalité, que l'individu considéré possède un flagelle (forme Mastigamoba), ou qu'il en soit privé (forme Dinamœba), le nombre 2 est la règle, qui ne souffre pour ainsi dire pas d'exceptions; mais pour se rendre compte de la chose, il faut, ou bien isoler et comprimer les individus, ou bien les examiner après coloration au carmin, et c'est ce que l'on n'a pas fait.

Un mot encore, à propos des aspérités si caractéristiques qui revêtent l'ectoplasme, ou couvrent les pseudopodes, et que l'on a décrites tantôt comme des "grains, granulations ", tantôt comme des « cils, spicules, baguettes» (Pl. 10, fig. 1 et 2).

Schulze, Leidy, GReEFF, et d'autres, ont fait ressortir l'analogie frappante que ces granulations montrent avec les Bactéries (Bacterium termo), mais personne n'ose se prononcer quant à leur signification réelle. BüTsCHLI, pourtant, est porté à y voir 
des bactéries véritables; Frenzel, Schneider, Goldschmidt sont contraires à cette idée. En 1905 (9, p. 590), après des observations minutieuses, j'étais arrivé à y voir à coup sûr des Bactéries vraies; mais en 1907, Goldschmidt, qui, sans doute, n'aura pas eu connaissance de mon travail, revient sur le sujet, et croit s'être bien assuré qu'il y a là, non pas des Bacilles, mais un produit spécial du plasma(DeutoplasmatischeBildungen), des granulations qui, dans certains cas, pourront être étirées en spicules ou baguettes; et ces particules, qu'il appelle «Klebkörner » ou grains agglutinants, auraient pour fonction d'assurer la marche, en fournissant à l'animal un appui solide sur le substratum; cette fonction, dit GoLDschnid, "serait alors la même que celle des clous aux souliers du montagnard». GoLdschmidT avait, en effet, remarqué que, pendant la marche, les «Klebkörner» se rassemblent à la partie postérieure de l'Amibe, et que l'animal se sert de cette extrémité comme d'un point d'appui, à partir duquel le corps est poussé en avant; puis ensuite cette partie postérieure fixée se détache et est attirée vers l'antérieure.

Cette hypothèse de GoLDschmidT, quant à la fonction de ces éléments minuscules, est certainement ingénieuse; mais il faut remarquer que, d'une part, notre Mastigamœba - comme toutes les Amibes, d'ailleurs, - un peu glutineuse par elle-même et surtout à sa partie postérieure, ne semble guère avoir besoin de ces « crampons » spéciaux, et d'autre part, que les individus qui s'en trouvent dépourvus marchent aussi bien que les autres.

Quant au fait que ces "Klebkörner » se rassemblent surtout en arrière, il n'y a là rien que de très naturel; toutes les Amibes, en définitive, ont leur surface revêtue comme d'une nappe extraordinairement mince de matière glutineuse, que l'animal, dans une marche rapide, laisse en quelque sorte derrière lui, comme s'il sortait d'un fourreau, et qui se rassemble, se tasse, à la partie postérieure, portant serrés les uns contre les autres 
les objets divers qui s'étaient collés un peu partout sur l'ectoplasme ${ }^{1}$.

Enfin, pour revenir à la nature même de ces grains, je ne puis que répéter ce que je disais en 1905, y ajouter même quelque chose, car en reprenant cette année (1909) leur étude, j’ai pu constater des cas de division de ces grains ou Bacilles, non seulement en deux ou en quatre articles, mais jusqu'en huit granulations, que j’ai pu voir une à une, allongées les unes derrière les autres. Dans le cas de partition en huit, le tout figurait un bâtonnet en spicule, de $3 \mu$ de longueur et de $1 / 2 \mu$ à peine en largeur . Dans la règle, sur un même individu, tous les Bacilles en étaient à peu près au même stade de développement. On sait que les différents observateurs qui se sont occupés de la question ont trouvé, soit des grains, soit des spicules ou baguettes, contradiction qui a paru étrange; mais nous pourrions, semble-t-il, expliquer aujourd'hui la chose en supposant la rencontre de Bacilles tantôt simples, tantôt composés et en chaînes.

Ces baguettes se voient généralement couchées de tout leur long, sur l'ectoplasme ou les pseudopodes, et c'est ainsi que, pour mon compte, je les ai toujours trouvées; mais elles peuvent, sans doute, se voir parfois relevées et comme piquées sur cet ectoplasme à la façon d'une épingle sur une pelote, en faisant avec cet ectoplasme un angle plus ou moins prononcé; ce qui explique également certaines divergences dans les descriptions des observateurs.

J'ai pu, cette année, colorer distinctement ces éléments bactéridiformes, par le bleu de méthyle.

${ }^{1}$ On peut faire à ce sujet des expériences intéressantes, et j'en ai relaté quelques-unes, en 1905, à propos de l'Amœba terricola (Arch. für Protistenk. Vol. 6, fasc. 2 , p. 178).

${ }^{2}$ En 1905 , j'indiquais les granulations sphériques comme mesurant $1 \mu$ environ, mais cette mesure n'était qu'approximative, et trop forte en réalité. Quant aux figures explicatives de ce même mémoire, il faut plutôt les considérer comme schématiques, les grains ayant été dessinés beaucoup trop volumineux relativement au pseudopode auquel on les voit fixés. 
Presque tous les observateurs ont mentionné la rencontre occasionnelle d'individus lisses, privés totalement des aspérités caractéristiques; cependant, j'ai pu m'assurer cette année que ces individus exceptionnels sont beaucoup plus rares qu'on ne l'a cru jusqu'ici. On trouve, il est vrai, toutes les transitions possibles entre les exemplaires couverts de grains par milliers et ceux qui n'en montrent que très peu ou pas du tout; mais, toutes les fois que j'ai pu isoler un de ces derniers, j'ai fini, après une compression graduelle et poussée très loin, par distinguer quelques Bacilles au moins.

C'est également dans un de ces individus lisses, très fortement comprimé, qu'un jour j'ai trouvé, en plein endoplasme, deux vacuoles spéciales, plutôt deux sacs, de $15 \mu$ de diamètre, pourvus d'une membrane bien nette, et à l'intérieur desquels couraient des milliers de grains, de $1 / 2$ \%. environ, en une danse échevelée qui certainement n'avait rien à faire avec un mouvement brownien. Sur le feuillet qui résume mes observations de ce jour-là, je n'ai pu m'empêcher d'écrire : « Ce sont là peut-être les spores (?) qui donneront les Bacilles caractéristiques. » Plus souvent il m'est arrivé de rencontrer ces grains ou « spores » simplement rassemblés en paquets dans certaines régions du plasma, comme formant par-ci par-là des nids, mais sans indication de membrane vraie; ils tourbillonnaient pourtant quelquefois (dans une vacuole alors), ou bien au contraire restaient immobiles, répandus au sein du plasma.

Ce sont là probablement ce que GoLdschnidt appelle des « lichtbrechende Körnchen » ou granulations réfringentes, et au sujet desquelles il s'exprime dans les termes suivants « : Ueber ihre chemische Natur vermag ich nichts auszusagen, halte aber eine Beziehung zu den Klebkörnern für wahrscheinlich. *

Quant à ces inclusions d'une autre nature encore, que GOLDsCHMIDT appelle des Bactérö̈des, et qui lui paraissent être bien certainement analogues aux bâtonnets ou Bactéries caractéris- 
tiques p. ex. des Pelomyxa, je ne me rappelle pas en avoir rencontré dans notre Mastigamibe, mais j'ai d'autant moins d'hésitation à leur attribuer la signification indiquée par GOLDSCHMIDT, que je les ai vus dans d'autres représentants du groupe, notamment dans cette Mastigella nitens dont il sera question plus loin.

\section{Mastigamœeba socialis $\mathrm{n}$. sp.}

(Pl. 10, fig. 3 et 4.$)$

GoLDSCHMIDT, dans un essai de classification qu'il ne considère que comme provisoire, mais qui répond suffisamment à l'état actuel de nos connaissances, a divisé les Mastigamibes en trois genres, Mastigamceba, Mastigina, Mastigella. Les deux premiers comprennent toutes les espèces dans lesquelles le flagelle prend naissance sur le noyau, le troisième concerne celles où il n'existe entre ces deux éléments aucune connection quelconque. Quant aux deux genres qui constituent le premier groupe, ils se distinguent l'un de l'autre par la faculté que possède l'un d'eux (Mastigamœba) de déployer de longs pseudopodes digités, cette faculté manquant au second (Mastigina), dont le corps n'éprouve guère que des changements en masse.

C'est alors au genre Mastigamœba qu'il faut encore rapporter un organisme trouvé à Feuillasse en novembre 1908, en compagnie de la Mastigamcba aspera, et auquel je proposerai d'appliquer cette épithète de socialis qui, il est vrai, s'applique avant tout à ses nombreux noyaux et vacuoles, qui semblent à première vue indiquer un organisme composé. Tout d'abord, on prendrait volontiers l'animal pour une Mastigamœba aspera; il en a la taille et l'apparence générale. Mais il suffit d'un moment pour y voir autre chose, pour y constater l'absence des Bacilles ou « Klebkörner » caractéristiques, et y reconnaître un plasma différent, plus délicat; le corps se voit en effet rempli, non plus de particules végétales grossières et de grains d'excrétion relativement 
volumineux, mais de parcelles nutritives plus petites et de myriades de granulations bien plus fines.

Lorsque l'animal se déplace dans le liquide, on le voit muni à sa partie antérieure d'un flagelle plus distinct que dans l'espèce précédente, quoique difficilement visible encore, et ce flagelle est ici d'une utilité bien plus évidente, car il entraîne l'Amibe dans une course assez rapide, en décrivant généralement de grands cercles.

En avant, le corps est pendant la marche étiré en un large prolongement de plasma pur et incolore; sur les côtés poussent et repoussent des pseudopodes, pâles, étroits, délicats, et tout le corps dans son ensemble éprouve des déformations assez fortes ${ }^{1}$; en arrière traînent et s'étalent des ìambeaux d'ectoplasme, déchiquetés ou étirés en pseudopodes d'un gris mat.

C'est là à peu près tout ce que montre un premier examen, sur un animal dans son état de nature, c'est-à-dire non comprimé; on n'y distingue en effet ni noyaux ni, dans la règle, de vésicules contractiles; quelquefois cependant l'une ou l'autre de ces dernières peut se montrer, lorsqu'elle se présente dans de bonnes conditions de visibilité.

Mais si nous isolons l'Amibe, et que nous la laissions se comprimer peu à peu sous le couvre-objet, nous arrivons à la connaître mieux, et nous y trouvons d'abord, au sein d'un plasma très clair, des myriades de grains, brillants, d'un jaune verdâtre, extrêmement petits ( $2 \mu$ environ pour la plupart); puis des proies généralement peu nombreuses, Chlorophycées vertes, petites Diatomées, enfermées souvent dans des vacuoles digestives. Nous voyons aussi des vésicules contractiles, éparses dans les couches superficielles du plasma, au nombre d'une douzaine, relativement très petites, paresseuses mais fonctionnant norma-

\footnotetext{
${ }^{1}$ La figure 4 représente un individu plutôt ramassé sur lui-même, et à pseudopodes relativement peu allongés.
} 
lement; enfin les noyaux, sur lesquels il faut nous arrêter un instant.

La Mastigamceba socialis, en effet, n'a pas un noyau comme la plupart des représentants de ce groupe, ni deux comme la $M$. aspera, mais elle en possède toujours un nombre assez considérable; dans un exemplaire de taille moyenne (50 $\mu$ à l'état arrondi et un peu comprimé), ce nombre était de 16, et sans doute arrive-t-il à un chiffre plus élevé dans les gros individus.

Ces noyaux, très petits ( 4 à $5 \mu$ environ), sont sphériques, et possèdent un gros nucléole central, d'un gris cendré, qu'une zone étroite de suc limpide sépare de la membrane nucléaire. Ils sont répandus un peu partout dans le plasma, mais c'est, naturellement, sur l'un d'eux seul, le plus antérieur, que s'insère le flagelle; la figure 3 montre cette insertion, telle qu'elle se voyait indiquée dans un individu examiné tout particulièrement sous ce rapport. Il semble que le flagellum soit, ici, pourvu d'un grain basal d'un volume tout à fait extraordinaire; mais cependant, il faut avouer que ces observations sont très délicates, et il pourrait y avoir erreur. Sur mes feuilles de notes, j'avais tout d'abord résumé ces observations dans les termes suivants : "Sur les cinq individus rencontrés, quatre montraient un flagellum, s'insérant sur l'un des noyaux, qu'il étire ou parfois semble diviser en deux. »

Que, pendant la marche, le flagelle étire la membrane nucléaire, comme nous l'avons vu pour la $M$. aspera, il n'y a rien là que de très naturel, mais qu'il se produise dans le noyau un étranglement en division quelconque, c'est là une éventualité déjà très peu vraisemblable, et probablement vaut-il mieux adopter la notion d'un grain basal volumineux, posé comme un bourgeon sur le noyau ${ }^{1}$. Ou peut-être le grain basal véritable serait-il invisible, perdu dans la masse d'un plasma spécial,

${ }^{1}$ C'est d'ailleurs ainsi que le ferait comprendre la fig. 3 , dessinée d'après le vivant, et qui semble être en contradiction avec la note relevée un peu plus haut. 
plasma ou bouton d'agglutination sur lequel nous aurons à revenir, à propos de Mastigina spicata et de Mastigella nitens.

Quant au flagelle lui-même, il est très délicat, mais nettement distinct, à peu près de même longueur que le corps, et relativement vif dans ses mouvements.

Comme on l'a vu plus haut, l'ectoplasme et les pseudopodes sont lisses, toujours absolument indemnes des aspérités ou Bacilles caractéristiques de la Mastigamœba aspera.

La taille est de 40 à $50 \mu$; mais probablement varje-t-elle dans des limites beaucoup plus considérables que ne l'indiquent ces chiffres. Cette espèce, en effet, ne s'est montrée représentée que par 5 individus, ce qui est trop peu pour des observations concluantes; mais deux au moins de ces individus, qui, d'ailleurs, étaient tous identiques en apparence, ont pu être suivis et étudiés tout au long.

\section{Mastigamoba auriculata n. sp.}

(Pl. 10, fig. 5ั à 7 ).

Les observations relatives à cette espèce remontent assez loin en arrière, à l'automne de 1902, mais il eût été intéressant de les contrôler, et je n'aurais pas songé à les publier encore si l'occasion ne s'offrait aujourd'hui d'elle-même de les joindre à ces pages consacrées aux Mastigamibes.

Ce Protiste n'a en effet été rencontré que deux fois, le 25 septembre à la Pointe à la Bise, sur les rivages du lac de Genève, puis le 16 octobre dans l'étang de l'Asile des Vieillards, et chaque fois un seul individu s'est montré; dans la dernière de ces localités même, l'Amibe n'a fait qu'apparaître un instant, courant au milieu des débris de toute sorte que recouvrait la lamelle, et tout ce que j'ai pu faire a été d'en prendre un croquis rapide (fig. 7), bien insuffisant, mais qui semble montrer pourtant que, malgré certaines différences de détail, nous avons, dans les deux cas, affaire au même organisme. 
Quant à l'individu trouvé à la Pointe à la Bise, et que représentent les figures 5 et 6 , il a pu être suivi tout au long; d'abord ramassé sur lui-même (fig. 6), il revêtit bientôt la forme que montre la figure 5, et - sauf les déformations spéciales dont il va être question - qu'il garda pendant un temps fort long, jusqu'au moment où il se perdit à son tour.

L'animal, pourvu d'un flagelle un peu plus long que le corps (ce dernier supposé dépourvu des longs pseudopodes postérieurs), très fin, visible par intermittences, battant vigoureusement et entraînant l'individu dans une course rapide, figurait une masse ovoïde, plus large en arrière, bourrée de proies et de particules de toute sorte, et aussi, surtout à sa partie postérieure, de gros grains d'excrétion verdâtres.

Près de l'extrémité antérieure se montrait le noyau, au-devant duquel l'ectoplasme se prolongeait en une nappe pâle et dégarnie d'inclusions quelconques, brusquement terminée en une troncature presque droite, anguleuse sur ses côtés, et c'est du milieu de cette bordure päle qu'on voyait partir le flagelle.

Ce dernier, sans aucun doute, devait pénétrer l'ectoplasme et aller s'appuyer au noyau, car il en est toujours ainsi dans les Mastigamibes lorsque, pendant la marche, le noyau se voit normalement rapproché de l'extrémité antérieure; et d'ailleurs, la figure 7 , où, dans le second exemplaire trouvé, ce fil interne s'est montré bien net, suffirait à le prouver; mais, il faut le dire, dans notre individu type ce détail m'a échappé.

Le noyau lui-même figurait une masse plutôt volumineuse, d'un bleu très pâle et pur; tout auprès, sur le côté, se voyait une vésicule contractile, bien distincte.

A la partie postérieure du corps, le plasma était étiré en un certain nombre (cinq, dans les deux individus rencontrés ${ }^{1}$ ) de

${ }^{1}$ La fig. 6 ne montre que quatre de ces prolongements; plus tard seulement il en a poussé un cinquième, visible dans la fig. 5, laquelle, en réalité, représente l'animal un instant plus tard. 
prolongements ou faux-pseudopodes, non ramifiés, très longs, qui trainaient en arrière, s'allongeant ou se raccourcissant parfois, mais lentement et dans une faible mesure.

Mais les éléments les plus caractéristiques étaient figurés par les prolongements latéraux, deux de chaque côté, qu'on pourrait appeler les bras (fig. 5, a), et les auricules (b). Les premiers, qui n'existaient, lors de la rencontre de l'animal, que comme deux moignons à gauche et à droite de l'extrémité antérieure (fig. 6) s'allongèrent, d'abord perpendiculairement aux côtés du corps, puis un peu rabattus en arrière, et finalement, ils prirent la forme indiquée par la figure 5. Mais pendant que se dessinaient les bras, on voyait se former, un peu plus en avant, et un de chaque côté également, deux nouveaux prolongements, que j'appellerai les « auricules », plus étroits, très courts, faisant eux aussi un angle avec le corps, mais dirigés cette fois plutôt en avant.

Or, cette forme, une fois atteinte, ne changea plus, pendant toute la durée de l'observation; ou plutôt, faudrait-il dire, les prolongements latéraux, bras et auricules, ne se déformèrent plus que pour se reformer immédiatement et à la même place.

En effet, tandis que les lambeaux digités postérieurs restaient à peu près inertes et ne faisaient que traîner en arrière, il n'en était pas de même des bras et des auricules, qui de temps à autre se déformaient, se raccourcissaient, jusqu'à se rétracter et disparaître sans laisser de traces; mais alors, ils repoussaient toujours, avec une certaine rapidité, et reprenaient leur forme et leur position respectives.

C'est là que se sont arrêtées mes observations sur ce Protiste qui me parait présenter un intérêt tout spécial par le fait que nous avons là, en somme, une Amibe, une Amibe nue, que ne recouvre aucune enveloppe, et qui montre pourtant un commencement de symétrie bilatérale.

Il eût été intéressant de pouvoir vérifier sur plusieurs individus les faits observés sur un seul; peut-être ces faits ne se 
seraient-ils pas toujours montrés les mêmes, et le second exemplaire, représenté par la fig. 7 et trouvé plus tard dans une autre localité, indique en effet certaines différences; mais tels qu'ils sont, ces faits ne manquent pas d'intérêt.

La Mastigamœba auriculata constitue une très petite espèce; l'exemplaire plus spécialement étudié mesurait $15 \mu$. séulement (non compris les pseudopodes); l'autre, plus grand, atteignait $25 \mu$.

Cet organisme, qui sans doute représente une espèce bien autonome, aurait peut-être quelque affinité avec la Mastigamœba eilhardi de BürgER ${ }^{1}$, dont Goldschmidt donne la diagnose suivante : «Ein grosses, kegelförmiges Pseudopod, in dessen Mitte die Geissel entspringt, und kleine kammförmige Pseudopodien am Hinterende. Grösse bis $80 \mu$.. » Mais cette Mastigamoba eilhardi est beaucoup plus grande, les prolongements postérieurs sont au contraire relativement très courts, et il n'est pas question des bras latéraux qui sont l'élément le plus caractéristique de la Mastigamcba auriculata. GoLDschmidT, enfin, la donne comme une Mastigella et non plus une Mastigamoba, par le fait que le flagelle s'y montre indépendant du noyau.

\section{Mastigina spicata n. sp.}

(Pl. 10, fig. 8, et Pl. 11, fig. 9 et 10.)

Le genre Mastigina concerne des Mastigamibes qui, tout en possédant un flagelle inséré sur le noyau, n'émettent pas de véritables pseudopodes digités, et gardent en tout temps, malgré des déformations évidentes, leur forme générale plus ou moins cylindrique ou ovoïde.

1 Estudios sobre Protozoas Chilenos de lagua dulce. Anali de la Universitad de Chile. 1906. ? (Il est probable que GoLdschmidt se trompe en donnant cette date; peut-être est-ce 1896 ?, une année que je n'ai pas pu me procurer; en 1906, je n'ai rien trouvé. 
Ce genre Mastigina comporte aujoud'hui 5 espèces, dont 2, $M$. chlamys Frenzel et $M$. setosa Goldschmidt ${ }^{1}$, possèdent en commun ce trait caractéristique, que le corps tout entier est revêtu d'une couverture serrée de soies fines et rigides.

Peut-être même faudrait-il ramener ce chiffre de 2 à l'unité, car en décrivant sa Mastigina chlamys, Frenzel n'a parlé que d'une enveloppe "striée », et il n'est pas certain que GoLDschmidT soit dans le vrai en se basant sur une erreur possible de Frenzel pour assimiler cette enveloppe à un revêtement de spicules.

En effet, Frenzel est assez catégorique dans ses affirmations; il parle d'une «peau», "Hautschichte», rayée en travers, d'une « substance striée très régulièrement, ou traversée de pores ». Il n'a, il est vrai, pas pu s'assurer absolument de la chose, mais, dit-il, "il me parait improbable qu'il y ait là une rangée de bâtonnets, car la bordure périphérique s'est toujours montrée continue, et non pas interrompue et ponctuée, comme elle devrait le paraître en présence de bâtonnets ou de cils ».

De plus, Frenzel a constaté sur certains individus la présence de prolongements spéciaux, rayonnants, de pseudopodes analogues à ceux des Héliozoaires, qui manquent dans la $M$. setosa. Toutes les figures, enfin, données par FrEnzeL montrent une enveloppe striée en travers, et non pas une bordure de cils.

En trouvant, au mois de septembre 1902, à la Pointe à la Bise, puis en mai 1903 au marais de Bernex, le Protiste flagellé que je viens décrire à mon tour, j'ai cru tout d'abord y voir cette Mastigina chlamys de FrenzeL; mais il m'a fallu bien vite renoncer à cette idée; plus tard, lorsque a paru le mémoire de

\footnotetext{
${ }^{1}$ D'après Goldschmidt, cette $M$. setosa aurait été vue, antérieurement déjà, par Schneider (11), qui l'aurait étudiée comme étant la Mastigamœba aspera de Schulze; Schneider, en effet, aurait pris les soies pour les aspérités caractéristiques de cette dernière espèce.
}

Rev. Suisse de Zool. T. 17. 1909. 
GoLdschmid, j'ai tenté de l'assimiler à la Mastigina setosa, mais c'est autre chose encore.

En effet, la $M$. setosa est beaucoup plus grande, plus allongée dans sa forme; le flagelle est relativement plus court; le noyau revêt une structure différente; la pellicule d'enveloppe, une « peau épaisse, fortement réfringente, à reflets jaunâtres » est d'une autre nature; les cils sont plus longs, plus fins, etc.

Passons maintenant à la Mastigina spicata.

Dans cette espèce, la forme est à peu près ovoïde, ou bien quelque peu allongée et rétrécie en avant. Pendant la marche, ou plutôt la nage, cette forme est sujette à certaines modifications, mais lentes et de peu d'amplitude; le corps se recourbe, par exemple, en point d'interrogation, ou s'allonge quelque peu, ou s'élargit en arrière, mais il garde en somme son apparence générale; les déformations les plus considérables sont causées par ces sortes de déchirures brusques, avec épanchements subits d'ondes successives qui se figent aussitôt en une large expansion de plasma.

Ce sont ces mêmes phénomènes, du reste, que GoLDschmidT a reconnus dans sa Mastigina setosa, et l'auteur dit à ce propos: « In seinen Einzelheiten stimmt der Prozess sehr gut mit der typischen Pseudopodienbildung überein, die RHUMBLER (1898) so schön von seiner Amœba limicola beschreibt ${ }^{1}$ ».

A la partie postérieure du corps, il se forme quelquefois, pendant la marche, une sorte de bordure frangée, ou de « houppe caudale », que l'animal traîne derrière lui.

Une grosse vésicule contractile, postérieure, vient de temps à autre faire saillie sur ce plasma caudal, lequel même se voit quelquefois recouvrir la vésicule d'une auréole complète de filaments pâles et serrés (fig. 10,a).

${ }^{1}$ C'est là ce que Rhumbler a appelé “ Fontainestrom ", un mode très original de progression que montrent certaines Amibes, A. limicola, undosa, fluida, etc., et que, soit dit en passant, j'avais déjà décrit tout au long dès l'année 1890 (Mém. Soc. Phys. Hist. Nat. Genève, tom. 31 no 2, pp. 68 à 70 ). 
Tout en avant, près du noyau, se voit également, dans la règle, une seconde vésicule contractile, qui fonctionne normalement.

A l'extrémité antérieure du corps, et tout près même du bord, dans un plasma très pur où l'on ne trouve d'autres éléments figurés que quelques très petits grains bleus, se voit logé le noyau, volumineux, pourvu d'une membrane nucléaire bien nette. La matière chromatique s'y montre sous la forme d'un nucléole unique, très gros, séparé de la paroi nucléaire par une zone de suc limpide.

GoLdschmid, à propos de sa Mastigina setosa, a décrit le noyau comme « ein kugeliges Bläschen, das an der Stelle der Geisselinsertion einen feinen schornsteinartigen Aufsatz hat, den man im Leben bei genauer Profilstellung des ganzen sehr schön sehen kann (fig. 47,a)». Il se pourrait bien que, dans la $M$. spicata, il existât quelque "prolongement en cheminée » de cette nature; à l'époque où j'examinais cet organisme, en 1903, je n'avais tout d'abord constaté, il est vrai, qu'un étalement bleuâtre, au pied du flagelle; mais, sur un nouvel examen, j'en étais arrivé au croquis que reproduit la figure $9, a$, où l'on voit un léger renflement au pôle antérieur du noyau; puis, un instant après, j’avais quelque peu corrigé mon dessin, en ajoutant à gauche et à droite de la base du flagelle deux petits traits qui vont rejoindre la membrane du noyau, et circonscrivent alors assez nettement une sorte de prolongement tubuliforme, lequel à son tour servirait de point d'attache à l'épanchement de plasma bleu (fig. 9, b). Ce pourrait alors être là la «cheminée » de GoLdschmidT, que l'auteur représente d'une manière à peu près analogue; mais il m'est impossible, en l'absence de toute note écrite, de me prononcer sur la signification réelle de mon croquis, et la mémoire me fait défaut à ce sujet. Par contre, pour ce qui concerne l'étalement à la base du flagelle, mes souvenirs sont plus précis, et je puis donner cet étalement comme certain. Seulement, ici encore, faut-il considérer cette masse bleuâtre 
comme représentant le grain basal étalé, ou ce grain est-il en réalité perdu dans cette masse spéciale, au travers de laquelle on ne le verrait plus ${ }^{1}$ ?

Le plasma de la Mastigina spicata, dans les individus rencontrés (en fort petit nombre, malheureusement, trois ou quatre), s'est constamment montré bourré de parcelles de nourriture, Diatomées, Chlorophycées, de grains d'excrétion, de gros corps clairs, aplatis, qui rappelleraient des leucites végétaux, de granulations brillantes, d'un vert bouteille, accumulées surtout en arrière, enfin, de particules de toute sorte, dont nous ne nous. occuperons pas plus au long.

Mais il nous faut maintenant considérer l'enveloppe, cette armature spéciale de spicules, qui semble ici différer assez fort de ce que l'on connaît ailleurs.

Nous n'avons plus, en effet, des « cils» ou des «soies » très fines, mais plutôt des spicules lamellaires, fusiformes, pointus aux deux bouts (fig. 10, b). Les plus petits, minces et courts, et qui sont probablement de formation plus récente, sont pâles, clairs, incolores, et se voient surtout à la partie antérieure du corps; puis ils deviennent plus granàs à mesure qu'ils se rapprochent de l'extrémité postérieure, plus vigoureux, en même temps qu'ils revêtent une teinte jaunâtre ou même brune, indice probable d'une nature chitineuse.

Ces spicules sont disposés sans grand ordre à la surface du

1 Goldschmint décrit et figure la chose d'une manière assez particulière : "Der Schornstein ist abgeschlossen durch eine Endplatte, die sich im Präparat etwas stärker färbt (fig. $47 b$ ) und dann leicht als vom Kern unabhängige Scheibe fälschlicherweise erscheinen kann, ein Eindruck, der in dem Fig. 41 zugrunde liegenden Präparat vorgetäuscht wurde. In der Mitte dieser Platte befestigt sich die Geisselwurzel und zeigt kurz vorher eine feine punktartige Anschwellung, die ebenfalls im Leben zu erkennen ist, eine Art Basalkorn (Fig. 47). „ Autrement dit, Goldschmidt aurait vu le grain basal libre, au-dessus de cette "plaque terminale , dans laquelle, pour mon compte, je crois voir cet étalement bleuâtre (colorable, en effet, par le carmin), propre à la plupart des Mastigamibes et dont il a été fait mention plus haut. 
corps, plus serrés dans certaines régions que dans d'autres, et surtout nombreux en arrière. Suivant toute apparence, ils sont susceptibles de changer leurs positions respectives. Ils sont piqués, en effet, comme des aiguilles, dans une sorte de membrane, non pas une vraie pellicule comme GoLdschnidt l'a décrit pour sa $M$. setosa, mais une substance spéciale, une nappe exsudée par le plasma, incolore ou très légèrement jaunâtre, et à laquelle, parfois, on peut vaguement reconnaitre un double contour. Mais cette membrane d'enveloppe est molle, plastique, les pointes des spicules peuvent s'y déplacer, et lorsque se produisent les jets de plasma caractéristiques, cette enveloppe se perce et semble se fondre, pour se reconstituer plus tard; elle serait peut-être, en somme, de même nature que celle de certains Cochliopodium, où elle ne représente qu'une pâte molle, susceptible de s'ouvrir en un point quelconque pour laisser passer un pseudopode ${ }^{1}$.

\section{Mastigina lacustris n. sp.}

(Pl. 11, fig. 11, 13, 14, 15.)

C'est encore au genre Mastigina qu'il faut rapporter un organisme rencontré au mois d'octobre 1909, dans le lac, aux environs de Genève; il vivait à 25 ou 30 mètres de profondeur, en compagnie de ces Rhizopodes caractéristiques des lacs d'origine glaciaire, et qui ne se retrouvent pas dans les marais.

${ }^{1}$ Peut-être faudrait-il assimiler l'Amœba pilosa de CASH à une Mastigamibe, et plus spécialement à la Mastigina setosa de Goldschmidt. Mais peut-être CAsH a-t-il aussi vu la Mastigina spicata; il dit en effet, à propos de certains individus rencontrés dansu ne station particulière: "The Fearnhead examples of A. pilosa presented some differences from the typical Chelford form. They were probably older individuals. The investiture was the same, but it was observed that the individual spicules were stouter, and many of them were darker in colour, assuming often a yellowish-brown hue, whilst the endoplasm of the animal was denser . 
Malheureusement, il n'en a été recueilli que deux exemplaires, et c'est sur l'un d'eux seul qu'est basée l'étude qui va suivre. Mais cet exemplaire a pu être étudié tout au long, d'abord au milieu des débris, puis isolé; et comme, d'autre part, le second individu, entrevu un instant puis perdu, s'est, en tout cas, dans un examen rapide, montré identique au premier, il n'y a pas lieu d'attendre, pour décrire cette espèce, une occasion qui probablement ne se présentera plus ${ }^{1}$.

Le corps, dans son ensemble, revêt une forme assez changeante qu'on peut appeler tantôt cylindrique, tantôt fusiforme, tantôt plus ou moins allongée-lobée, recourbée, etc.; les déformations s'y montrent donc assez fortes, mais jamais on ne constate de déployement de bras véritables, digités ou pointus.

La teinte générale, d'un bleu délicat d'aigue-marine, est très claire et pure, comme on la trouve souvent dans les organismes des lacs profonds et limpides.

Ce qui frappe, au premier abord, c'est la ligne particulièrement franche et nette qui délimite le corps; il semble qu'il y ait là une enveloppe, très réfringente; et en effet, cette enveloppe existe, on y peut-reconnaître un double contour; le plasma s'est durci à la périphérie, et a acquis, temporairement au moins, la consistance d'une membrane.

Cependant, cette pellicule hyaline ne se voit pas sur la périphérie tout entière du corps; il existe deux régions où, pendant la marche, elle manque absolument: la région antérieure, et celle qui lui est opposée à l'autre extrémité du corps. La première, en effet, préside avant tout à la locomotion qui, dans cette espèce, se fait en «fontaine *, par une série d'ondes et de vagues qui déferlent coup sur coup, soit à l'extrémité même du corps, en avant du noyau, soit tout près, sur l'un des côtés.

${ }^{1}$ Dans ce second individu, le noyau n'a pas été aperçu, par le fait que l'animai était, par sa partie antérieure, caché sous les débris. 
Dans ce dernier cas, il y a changement de direction, le plasma se figeant bien vite et à chaque rague produite, l'animal faisant ainsi, par sa partie antérieure, un angle plus ou moins prononcé avec sa direction primitive. C'est ce que peut aider à comprendre la fig. 14, où les lignes pointillées indiquent deux vagues successives.

Quant à l'extrémité postérieure, elle est représentée par un plasma particulier, cendré, moins aqueux, susceptible, comme dans les Amibes en général, de s'étirer en prolongements ou lambeaux. Dans la fig. 13, on voit toute cette partie caudale former une agglomération bizarre, nettement distincte du corps proprement dit, lequel à ce moment-là formait une masse à peu près ovoïde; l'animal était en train de s'allonger pour la marche, et bientôt après prit l'apparence représentée par la fig. 14, puis enfin celle de locomotion rapide, que montre la fig. 15.

Dans la Mastigina lacustris, nous trouvons un plasma si fortement vacuolisé, que les vacuoles remplissent le corps tout entier, sauf l'extrémité antérieure où elles ne pénètrent jamais ; l'extrémité postérieure, cependant, en est également quelquefois dégarnie, mais en général on les y trouve, pénétrant même jusque dans les prolongements caudaux (fig. 15).

Ces vacuoles sont assez serrées les unes contre les autres pour se toucher par leurs bords, mais elles restent parfaitement rondes, sans se déformer mutuellement. Elles ne sont pas contractiles, pas sujettes à des modifications quelconques, mais pourtant il existe sans doute une vésicule contractile vraie, car, tout près et en arrière du noyau, on pouvait voir une vacuole tout particulièrement volumineuse, qui tranchait nettement sur toutes les autres, et à laquelle il serait difficile de refuser l'attribution indiquée plus haut; mais je ne l'ai pas vue fonctionner.

En suite de cette vacuolisation générale, l'endoplasme proprement dit se réduit, en somme, aux mailles qui séparent les vésicules les unes des autres, un plasma hyalin, où l'on voit, le 
plus souvent par groupes de deux ou de trois, dans les interralles que laissent entre elles les vacuoles, un grand nombre de petits grains vert-olive, de 2 à $3 \mu$ de diamètre, brillants et purs, et qui sont promenés lentement de côté et d'autre pendant les déformations de l'animal.

Ces grains constituaient, dans les deux individus rencontrés, le seul élément figuré que renfermât le corps; on n'y trouvait pas une parcelle de nourriture.

Le noyau, très rapproché de l'extrémité antérieure, est nettement visible, au milieu du plasma pâle où ne pénètrent ni grains ni vacuoles; il est sphérique, et de $8 \mu$ en diamètre; à certains moments, pendant la marche, on le voit légèrement étiré en avant.

La substance chromatique y est représentée par un seul nucléole, très volumineux, séparé de la membrane nucléaire par une zone étroite de suc limpide. Ce nucléole, dans l'individu examiné, renfermait une demi-douzaine de vacuoles rondes, bien nettes, l'une d'elles relativement très grande, et pendant tout le temps qu'a duré l'observation, ces vacuoles sont restées à la même place, sans éprouver aucune modification d'apparence.

Le flagelle est très court, arrivant au quart seulement de la longueur de l'animal (supposé allongé en Limace); il est relativement bien distinct, droit, presque rigide, et, quoique secoué de temps à autre de quelque faible vibration, il ne semble être d'aucune utilité en tant qu'organe de locomotion; plutôt, lorsqu'on le voit pointer droit devant lui, pendant que l'animal rampe, et pour ainsi dire coule, sur le substratum, le prendrait-on volontiers pour un organe de tact.

Ce flagelle est inséré sur la membrane nucléaire par une sorte de bouton bleuâtre, qui, d'abord conique, va s'étalant sur les côtés, en une nappe d'abord assez épaisse, puis s'amincissant toujours plus, et qui couvre ainsi le noyau sur plus du quart de sa superficie (fig. 11). 
La Mastigina lacustris représente une assez grande espèce, la plus grande, jusqu'ici, du genre, après la $M$. setosa. Sous la forme indiquée par la fig. 15, l'animal atteignait $108 \mu$.

Comme apparence générale, elle rappellerait la Maistigina paramylon de FrenzeL, plus petite et munie d'un long flagelle, ou bien encore la Mastigina hyla (Tricholimax hylæ Frenzel), de même volume à peu près, et dont le flagelle, de $10 \mu$ seulement de longueur, reste lui aussi à peu près inerte et a été comparé par Frenzel à un « cirre ». On pourrait même être tenté d'assimiler l'un à l'autre ces deux organismes, n'était que la $M$. hylæ est complètement privée, soit de vésicule contractile, soit même de vacuoles ordinaires.

Cette $M$. hylæ a été trouvée dans le rectum de petits têtards verts, état jeune d'une Rainette de la République Argentine. Au fond du Léman, où l'on ne connaît de Batraciens d'aucune sorte, la Mastigina lacustris vit sans doute parfaitement libre.

\section{Mastigella nitens $\mathrm{n}$. sp.}

(Pl.11, fig. 12, 16, 17.)

En étudiant, au mois d'août dernier, la Mastigamoba aspera, dans une récolte qui provenait de Feuillasse, je remarquai dès les premiers jours certains individus, relativement clairs, réfringents sur leur bord, mais que je ne songeai pas tout d'abord à séparer de l'espèce à ce moment en observation. Mais bientôt, il me fallut reconnaître que ces deux Mastigamibes différaient radicalement l'une de l'autre, et que la possession d'un flagelle tout à fait indépendant du noyau faisait rentrer le nouveau venu dans le genre Mastigella. Un instant, j'ai voulu y voir la $\boldsymbol{M}$. vitræa de GoLDSCHMIDT, mais il y a là autre chose encore.

Notre Mastigelle est d'assez forte taille, 80 à $90 \mu$. et plus encore, plutôt large, vigoureuse, sujette à des déformations considérables, mais en somme étalée et aplatie plutôt que cylindrique. Le corps est tout entier revêtu d'une enveloppe véritable, 
un simple durcissement de l'ectoplasme à sa périphérie, sans doute, et d'essence fugitive, susceptible de se fondre ou de se reformer suivant le besoin, mais qui n'en représente pas moins en fait une pellicule, opalescente sur ses bords, extraordinairement mince, mais réelle, car si par compression brusque on fait éclater l'individu, on peut obtenir cette pellicule à l'état isolé, comme un sac vide et plissé. C'est à la partie antérieure et sur les côtés que cette pellicule se voit le plus nettement, et elle paraît manquer à la partie postérieure du corps lorsque ce dernier, pendant la marche, s'allonge en lambeaux filamenteux et déchiquetés.

La masse proprement dite du corps, arrondie et épaisse, remplie de proies de toute sorte, Diatomées et autres, bourrée de grains brillants plus nombreux, mais beaucoup plus petits, que ceux de la Mastigamoba aspera, se voit généralement entourée d'une bordure d'ectoplasme très pur, très clair, très mobile, qui se transforme continuellement, se répand de tous les côtés, en vagues, en lobes, en bras, lesquels empiètent les uns sur les autres, se mélangent, etc., et tout cela sans jamais perdre leur reflet brillant qui dénote l'existence de la pellicule caractéristique. Par-ci par-là, cependant, se forment des pseudopodes plus longs, digités, quelquefois bifurqués, et qui paraissent être dépourvus de pellicule; à la partie postérieure surtout, où les prolongements sont quelquefois très longs et glutineux.

Sur l'un ou sur l'autre des lobes d'ectoplasme, et le plus souvent au sommet d'un petit prolongement spécial, auquel il est fixé, presque à la surface, par un corpuscule basal sphérique et très petit, se montre le flagellum, très mince, difficilement visible, qui se promène dans le liquide ambiant en longs mouvements amples et gracieux. Pendant la marche, il est le plus souvent en avant, mais cela n'est pas même nécessaire, et il change volontiers de place, entraîné avec son grain basal dans les vagues de l'ectoplasme. Il est probable qu'ici comme dans tant d'autres Mastigamibes, le flagelle ne sert pas à grand'chose, sauf sur 
les individus tout jeunes; chez les adultes, il n'atteint guère plus de la moitié de la longueur du corps, fonctionne d'une manière paresseuse, et est si délicat, que planté sur l'ectoplasme on pourrait le comparer à la ficelle qui tient un cerf-volant, en supposant cette ficelle coupée un peu en avant de l'appareil.

Ici comme dans la Mastigamoba aspera, beaucoup d'individus se montrent dépourvus de flagelle, mais en cherchant bien, on finit généralement par le découvrir; cependant, plusieurs fois aussi, sur des exemplaires isolés, comprimés, examinés longuement, il m'a été impossible d'en trouver trace (fig. 16).

J'ai cherché à voir comment disparaît le flagelle, en isolant les individus, en les laissant se comprimer graduellement sous le couvre-objet; et toutes les fois que j'ai pu mener l'expérience jusqu'au bout, c'est-à-dire sans perdre le flagelle de vue en suite d'une fatigue de la rétine, j'ai vu les mêmes faits se succéder : le flagelle bat d'une manière toujours plus paresseuse, les ondulations se font plus lentes et plus rares; de longues minutes s'écoulent, pendant lesquelles il reste immobile; puis il ondule. une fois ou deux encore, et tout d'un coup... on ne voit plus rien, plus rien que le grain basal, qu'on peut suivre quelque temps encore, jusqu'au moment où il se confond avec d'autres granulations ou micelles très petites répandues dans le voisinage.

L'anéantissement du flagelle provient le plus souvent de ce que ce flagelle s'est renversé, couché brusquement et tout d'une pièce sur l'ectoplasme, pour se fondre avec ce dernier; d'autres fois le fil, en plein liquide, semble s'émietter brusquement en un chapelet de grains qui disparaissent aussitôt.

Notons aussi que le flagelle peut à l'occasion s'enrouler sur lui-même à son extrémité, y formant une petite boucle ${ }^{1}$; d'où l'on peut inférer que, ici comme par exemple dans la Multicilia

1 Cette observation spéciale a été faite, il faut le dire, sur la Mastigamœba aspera, où mes études concernant le flagelle ont donné des résultats absolument identiques à ceux fournis par la Mastigella nitens. 
lacustris, où la chose a été étudiée plus à fond ${ }^{1}$, ce flagelle va probablement bientôt se couper au-dessous de la boucle, et se raccourcir d'une longueur plus ou moins forte.

Dans cette espèce, la masse générale du corps est assez compacte, et les granulations, proies et particules qui bourrent le plasma rendent les observations difficiles; cependant, il est deux éléments qu'on distingue presque toujours sans peine, quelle que soit l'opacité du corps; ce sont la vésicule contractile et le noyau.

La vésicule contractile est en effet très grande, et, en corrélation sans doute avec ce volume même, sujette, dans ses pérégrinations au sein du plasma, à des déformations fortes et quelquefois bizarres. On la trouve tantôt ici, tantôt là, sur les bords du plasma central; mais, dans le cas d'une marche rapide, èlle se voit le plus souvent en arrière, et peut faire sur le milieu ambiant une très forte saillie (fig. 16), pour se vider au dehors de la manière habituelle, et se reformer ensuite à l'endroit où elle avail éclaté. Souvent, au lieu d'une seule vésicule, il y en a plusieurs, assez volumineuses même, et qui probablement finiront par se réunir en une seule.

On trouve également, par-ci par-là dans le plasma, quelques petites vacuoles rondes, puis quelquefois des Bactéries ou baguettes, allongées et divisées en articles, et qui parfois forment des agglomérations, des nids, dans telle ou telle région du plasma.

Ces Bactéries n'ont rien à faire avec les granulations ou Bacilles, "Klebkörner » de Goldschmidt, que nous avons vus sur les pseudopodes de Mastigamoba aspera, et qui du reste manquent absolument dans notre Mastigella; par contre, elles sont tout à fait semblables aux Bactéries caractéristiques du genre Pelomyxa.

Il nous reste à parler du noyau, qui dans cette espèce est d'un volume tout à fait extraordinaire, arrivant à peu près au tiers

${ }^{1}$ Revue Suisse de Zool. Tome II, fasc. I, 1903, p. 138. 
du diamètre de l'animal (ce dernier supposé ramassé sur luimême).

Il est sphérique, ou bien se montre légèrement elliptique, par suite d'un aplatissement aux pôles; au premier abord, on le distingue comme une large tache grisâtre, bien apparente et qu'on prendrait pour un objet étranger.

Ce noyau (fig. 17) revêt une structure toute particulière, et qui permettra toujours de reconnaître facilement cette espèce : la masse chromatique, en effet, y est rassemblée, sous forme de granulations ou poussières extrêmement ténues, tout entière sous la membrane nucléaire, en une seule couche continue, d'un gris mat, mince, mais d'une épaisseur inégale suivant la région considérée. Sur un noyau isolé, puis prudemment comprimé, où cette couche grise peut se voir, non plus seulement par la tranche et sous la forme d'un anneau, mais aussi perpendiculairement à son épaisseur, on se trouve en présence d'une sorte de réseau, à mailles plus claires et à trame plus foncée, les espaces clairs indiquant les régions où la couche est moins épaisse. Quant à l'espace central, que circonscrit la matière chromatique, il est rempli par le suc nucléaire, presque limpide, piqueté par-ci parlà de tout petits points gris, lesquels représentent des parcelles nucléolaires, nageant pour ainsi dire en pleine eau et emportées de côté et d'autre.

Le noyau possède une membrane, qu'on peut même isoler par un coup sec porté sur le cover; cette membrane se déchire alors, en expulsant son contenu; mais elle est extrêmement mince, et la matière chromatique plaque si bien sur sa paroi interne, qu'on ne la voit pas, et que c'est la couche chromatique elle-même qu'on serait tenté de considérer comme la membrane nucléaire ${ }^{1}$.

${ }^{1}$ Le cas est ici à peu près celui de l'Actinophrys sol, dont le noyau a beaucoup d'analogie avec celui qui vient d'être décrit, et où la vraie membrane nucléaire est, d'après moi, longtemps restée ignorée (v. Penard, Héliozoaires d'eau douce, Genève 1904, p. 103). 
La Mastigella nitens s'est rencontrée, au mois d'août, puis en septembre de cette année (1909), au marais de Feuillasse, où elle n'était pas très rare; mais il fallait de la patience pour en obtenir quelques exemplaires, parce qu'au lieu de se montrer, comme tant de Rhizopodes, volontiers à la surface du dépôt au fond du bocal, elle semble avoir une tendance prononcée à aller se cacher au sein du dépôt même.

Nous avons dit que, dans ce petit marécage de Feuillasse, la Mastigella nitens se trouvait mêlée à la Mastigamoba aspera; et c'est aussi là, on l'a vu plus haut, que s'est rencontrée la Mastigamcba socialis; trois espèces vivant en compagnie dans un espace très restreint. Le fait peut paraître surprenant en lui-même, car les Mastigamibes sont en somme des organismes rares; mais il ne faut pas oublier qu'il n'en est pas des Protozoaires comme des animaux supérieurs, dans lesquels la lutte pour l'existence est avant tout (en l'absence de l'homme) une lutte pour la nourriture; et, les formes proches parentes ayant les mêmes besoins, la plus faible finit par y laisser la place à la plus forte. Ici, les choses sont différentes; la nourriture, sous la forme habituelle d'organismes végétaux minuscules presque toujours très nombreux, suffit dans la règle plus que largement à tous, et les conditions de réussite dépendent avant tout du milieu ambiant, de la nature de l'eau, peut-être de la qualité de la nourriture, etc., et l'on peut dire que sous ce rapport beaucoup de Rhizopodes, par exemple, sont beaucoup plus exigeants qu'on ne le pense; mais, étant donné un milieu favorable, ce milieu se voit vite envahi, et les espèces les plus proches ayant vraisemblablement les mêmes besoins, finissent par se trouver réunies. 


\section{LISTE BIBLIOGRAPHIQUE}

1. Archer, W. Résumé of recent contributions to our knowledge of Freshwater Rhizopods. Quart. Journ. Mic. Sci. new ser. Vol. 17, 1877.

2. Blochmann, F. Kleinere . Vittheilungen über Protozoen. Biolog. Centralbl., Vol. 14, 1894.

3. Cash, J. The British Freshwater Rhizopods and Heliozoa, publ. by the Ray Society, Vol. 1, 1905.

4. Frenzel, J. Untersuchungen über die milir. Fauna Argentiniens. I. Die Protozoen, Abt. 1 u. 2, Bibliotheca Zoologica, Hft. 2, 1892.

ร. Goldschmidt, R. Lebensgeschichte der Mastigamöben. Arch. f. Protistenk. Festband R. Hertwig, 1907.

6. Greef, R. Sludien über Protozoen. Sitzungsber. d. Ges. Naturw. Marburg, No $3,1888$.

7. Leidy, J. Freshwater Rhiz. of North America. Rep. U. S. Geol. Survey of the Territ., Vol. 12, 1879.

8. Penaro, E. Faune Rhizopodique du Bassin du Léman. Genève, 1902.

9. Penard, E. Notes sur quelques Sarcodinés. $1^{\text {re }}$ part. Revue Suisse Zool. Tom. 13, fasc. 3, 190 əั.

10. Rhumbler, L. Physikalische Analyse von Lebenserscheinungen in der Zelle. Arch. f. Entwicklungsmech., Vol. 7, 1898.

11. Schneider, K. C. Plasmastruktur und Bewegung bei Protozoen und Pflanzenzellen. Arb. Zool. Instit., Wien, Vol. 16, 190 .

12. Schulze, F.-E. Rhizopodienstudien. Arch. f. mikrosk. Anat. Vol. 11, $18 \div 5$. 


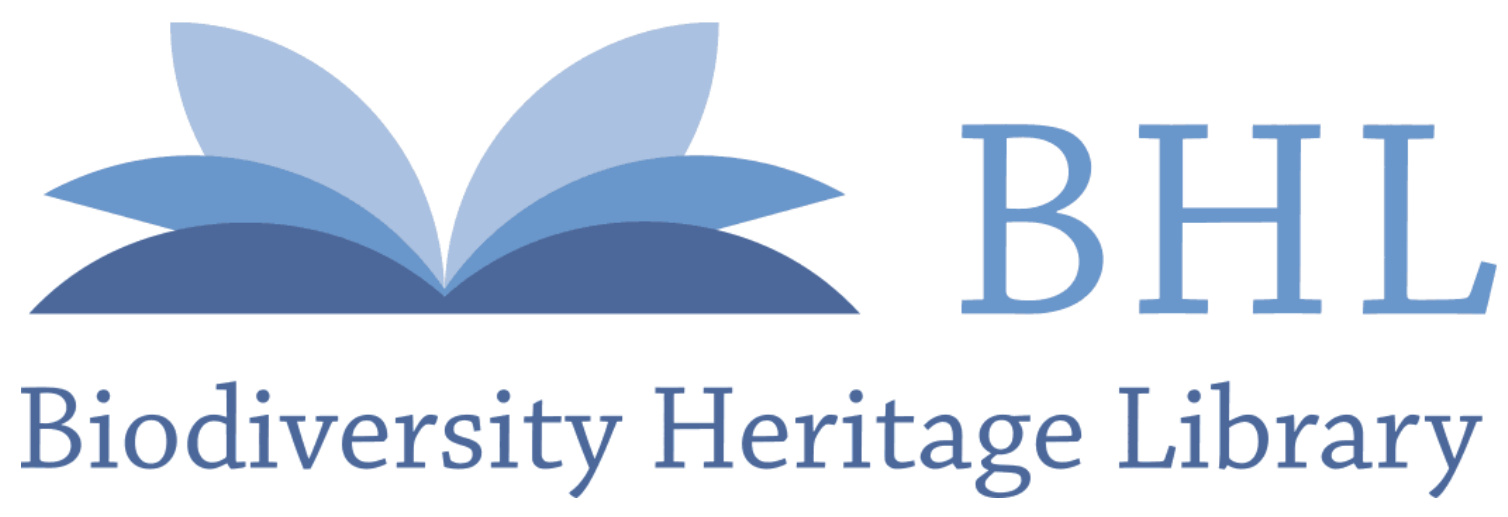

Penard, Eugène. 1909. "Sur quelques Mastigamibes des environs de Genève." Revue suisse de zoologie 17, 405-439. https://doi.org/10.5962/bhl.part.75199.

View This Item Online: https://www.biodiversitylibrary.org/item/40663

DOI: https://doi.org/10.5962/bhl.part.75199

Permalink: https://www.biodiversitylibrary.org/partpdf/75199

\section{Holding Institution}

MBLWHOI Library

Sponsored by

MBLWHOI Library

\section{Copyright \& Reuse}

Copyright Status: NOT_IN_COPYRIGHT

This document was created from content at the Biodiversity Heritage Library, the world's largest open access digital library for biodiversity literature and archives. Visit BHL at https://www.biodiversitylibrary.org. 